\title{
EDITORIAL
}

\section{EL CUIDADO INFORMAL COMO OBJETO DE ESTUDIO}

\author{
INFORMAL CARE AS AN OBJECT OF STUDY
}

\author{
Prof. Dra. Viviane Jofré Aravena \\ VICEPRESIDENTA \\ Revista Ciencia y Enfermería
}

\begin{abstract}
T a definición y medición del cuidado es un tema en debate. Los límites del cui$\triangle$ dado son difíciles de demarcar en términos de qué se hace, a quién, dónde y durante cuánto tiempo. Esta complejidad plantea dificultades a la hora de dimensionar su magnitud. La mayoría de las investigaciones toman como punto de partida un tipo de persona dependiente, a partir de la cual identifican a sus cuidadores. Sin embargo, el cuidado informal ocurre en todas las edades y para un amplio espectro de condiciones (1). En sentido amplio, todos somos, en algún momento de nuestras vidas, beneficiarios del sistema informal, y muchos de nosotros y nosotras somos o seremos cuidadores (2).
\end{abstract}

En cualquier situación, es relevante considerar que los cuidados informales siempre implican una relación afectiva definida entre el proveedor y la persona receptora de cuidados por el que no se recibe remuneración económica.

Gran parte de la investigación sobre cuidados informales se ha centrado en el análisis de las consecuencias que tiene sobre la/el cuidadora/or el hecho de atender la salud de sus familiares y el impacto y la angustia correspondiente que le produce asumir los cuidados de un familiar. Este proceso de percepción del impacto de los cuidados y la angustia (distress) que se le asocia es lo que se denomina sobrecarga de la cuidadora/or (3).

La sobrecarga que produce la tarea de cuidar ha tendido a estudiarse con un enfoque epidemiológico de riesgo y a través de modelos de manejo de estrés y de afrontamiento, olvidando considerar el aspecto positivo de esta actividad (4). Esto produce la impresión de que cuidar es una clase de enfermedad, las personas que cuidan son susceptibles de padecerla y de fallecer a causa de ella. 
El cuidado familiar en sí mismo no produce la morbilidad y mortalidad reportada, más bien se puede argumentar que son las condiciones bajo las que se da este cuidado. Asuntos étnicos, culturales, de género y sociales intervienen en esta carga (5-7), por lo tanto la carga del cuidado es un asunto más de desigualdad en salud.

Por lo anterior es urgente que los profesionales sanitarios visualicen las necesidades de las cuidadoras y les ofrezcan recursos y alternativas. Desde el nivel de planificación y gestión de servicios, ofertando servicios accesibles y adecuados de apoyo. Y desde el nivel de políticas de salud, en el que se aborden estrategias para eliminar la situación de desigualdad de género existente entre hombres y mujeres en relación al cuidado de salud de las personas de la comunidad, que lo necesi$\tan$.

Todo lo anterior no puede ser enfrentado desde la realidad, si para ello no se muestran las evidencias desde la cuantía del fenómeno y develando la esencia que esto significa para las personas involucradas.

\section{REFERENCIAS}

1. Arriagada I. La Crisis del Cuidado en Chile.Proyecto de CEM-Chile e INSTRAW "Construyendo redes: Mujeres latinoamericanas en las cadenas globales de cuidado". [citado julio 2011]. Disponible en: http://www.cem.cl/proyectos/ documentos/Arriagada_crisis.pdf.

2. García-Calvente MM, Mateo-Rodríguez I, Maroto-Navarro G. El impacto de cuidar en la salud y la calidad de vida de las mujeres. Gac Sanit 2004;(Supl 2):83-92.

3. García-Calvente MM, Mateo Rodríguez I, Gutiérrez Cuadra P. Cuidados y cuidadores en el sistema informal de salud: investigación cuantitativa. Granada: Escuela Andaluza de Salud Pública, 1999.

4. Jeon Y, Brodaty H, Chesterson J. Respite care for caregivers and people with severe mental illness: literature review. J Adv Nurs. 2005;49:297-306.

5. Hirst M. Hearts and minds: the health effects of caring. Heslington, York: Social Policy Research Unit, University of York; 2004.

6. Pinquart M, Sörensen S. Ethnic differences in the caregiving experience: implications for interventions. Geriatr Aging. 2005;8(10):64-66.

7. Grinyer A. Caring for a young adult with cancer: the impact on mother's health. Heal Soc Care Community. 2006;14: 311-18. 